\title{
The Method of Targeting a Group of Fighters, Taking Into Account the Nature of the Tasks they Solve
}

\author{
Denis V. Zakomoldin*a, \\ Alexander V. Bogdanov ${ }^{\mathbf{a}}$ and Vitaly A. Bulov \\ ${ }^{a}$ Military Academy of Aero-Space Defence \\ named after the Marshal of Soviet Union G. K. Zhukov. \\ Tver, Russian Federation \\ ${ }^{b}$ Military unit 15650 \\ Akhtubinsk, Russian Federation
}

Received 14.03.2021, received in revised form 10.05.2021, accepted 30.08.2021

\begin{abstract}
In this article, in the interests of improving the effectiveness of group actions of fighters, the method of targeting a group of fighters as part of a strike fighter, a cover fighter and an information support fighter at the $\mathrm{VC}$, taking into account the tactical purpose of each fighter in the group, is synthesized, and the characteristics of this method are evaluated on the basis of modeling. Within the framework of the synthesis, a local quadratic quality functional is defined that allows taking into account the accuracy of the guidance of the strike fighter, which is aimed at the zone of possible launch of airto-air missiles, the accuracy of maintaining the parameters of the combat order by the cover fighter relative to the strike fighter, the accuracy of maintaining the parameters of the combat order by the information support fighter relative to the cover fighter, and the cost-effectiveness of control signals. The list of meters that are necessary for the implementation of the synthesized method is determined, which indicates its practical feasibility.
\end{abstract}

Keywords: guidance method, control, strike fighter, information support fighter, cover fighter, group actions of fighters.

Citation: Zakomoldin D. V., Bogdanov A. V., Bulov V. A. The method of targeting a group of fighters, taking into account the nature of the tasks they solve, J. Sib. Fed. Univ. Eng. \& Technol., 2021, 14(7), 747-762. DOI: 10.17516/1999-494X-0345

(C) Siberian Federal University. All rights reserved

This work is licensed under a Creative Commons Attribution-Non Commercial 4.0 International License (CC BY-NC 4.0).

* Corresponding author E-mail address: denjuga68@yandex.ru 


\title{
Метод наведения группы истребителей \\ с учетом характера решаемых ими задач
}

\author{
Д. В. Закомолдин ${ }^{\mathrm{a}}$, А. В. Богданов ${ }^{\mathrm{a}}$, В. А. Булов ${ }^{0}$ \\ ${ }^{a}$ Военная академия воздушно-космической оборонь \\ им. Мариала Советского Союза Г. К. Жукова \\ Российская Федерация, Тверь \\ ${ }^{\sigma} \mathrm{B} / \mathrm{u} 15650$ \\ Российская Федерачия, Ахтубинск
}

\begin{abstract}
Аннотация. В данной статье, в интересах повышения эффективности групповых действий истребителей, синтезирован метод наведения группы истребителей в составе ударного истребителя, истребителя прикрытия и истребителя информационного обеспечения на ВЦ, учитывающий тактическое назначение каждого истребителя в составе группы, а также оценены характеристики данного метода на основе моделирования. В рамках синтеза определен локальный квадратичный функционал качества, позволяющий учесть точность наведения ударного истребителя, осуществляющего наведение в зону возможного пуска ракет класса «воздух-воздух», точность поддержания параметров боевого порядка истребителем прикрытия относительно ударного истребителя, точность поддержания параметров боевого порядка истребителем информационного обеспечения относительно истребителя прикрытия и экономичность сигналов управления. Определен перечень измерителей, которые необходимы для реализации синтезированного метода, что свидетельствует о его практической реализуемости.
\end{abstract}

Ключевые слова: метод наведения, управление, ударный истребитель, истребитель информационного обеспечения, истребитель прикрытия, групповые действия истребителей.

Цитирование: Закомолдин, Д. В. Метод наведения группы истребителей с учетом характера решаемых ими задач / Д. В. Закомолдин, А. В. Богданов, В. А. Булов // Журн. Сиб. федер. ун-та. Техника и технологии, 2021, 14(7). С. $747-762$. DOI: $10.17516 / 1999-494 X-0345$

\section{Введение}

Анализ особенностей военно-технического противоборства в воздушно-космической сфере последних десятилетий свидетельствует о существенном возрастании роли истребительной авиации [1]. Результатом применения истребительной авиации является уничтожение воздушных целей (ВЦ) противника. Эффективный способ поражения ВЦ противника - применение ракет класса «воздух-воздух». Для применения данных ракет необходимо предварительное наведение истребителя в зону возможного пуска ракет (ЗВПР) класса «воздух-воздух». С учетом того, что это справедливо для обеих конфликтующих сторон, превосходство над противником может быть достигнуто за счет обеспечения скрытного наведения истребителя в ЗВПР класса «воздухвоздух». Причем исходя из характеристик современных бортовых радиолокационных систем (БРЛС) истребителей, по возможности одновременного обслуживания большого количества ВЦ противника $[2,3]$ для завоевания превосходства над противником достаточно наведения одного из истребителей группы в ЗВПР и создания условий для применения им оружия. Факт наведения истребителя в ЗВПР может быть обнаружен посредством применения средств активной и пассивной локации, а именно БРЛС и станций РТР. Таким образом, в интересах обеспечения скрытного наведения истребителя в ЗВПР необходимо противодействовать БРЛС и станциям 
РТР противника. Одним из перспективных вариантов снижения возможностей БРЛС противника является ее радиоэлектронное подавление. Снижение возможностей средств РТР может быть достигнуто за счет снижения мощности излучения [4-6] или полного выключения БРЛС истребителя, осуществляюего наведение в ЗВПР. Групповые действия истребителей позволяют осуществить противодействие средствам активной и пассивной локации за счет распределения задач в составе группы. В частности, назначения в группе ударного истребителя (УИ), задачей которого является наведение в ЗВПР класса «воздух-воздух», с целью последующего поражения воздушных целей (ВЦ) с выключенной БРЛС в интересах противодействия станциям РТР. Определения истребителя прикрытия (ИП), задачей которого служит прикрытие ударного истребителя, посредством постановки активных помех БРЛС противника, включенной на излучение станцией активных помех (САП), и истребителя информационного обеспечения (ИО), задача которого заключается в формировании оценок параметров движения ВЦ противника и выдаче данной информации ударному истребителю для эффективного его наведения в ЗВПР.

Назначение ударного истребителя целесообразно осуществлять по критерию минимального расстояния до ВЦ противника. Данный истребитель осуществляет наведение в ЗВПР класса «воздух-воздух» одним из известных методов наведения [7-11].

Истребитель прикрытия с работающей на излучение САП должен осуществлять поддержание параметров боевого порядка, причем чем ближе ИП находится к объекту прикрытия УИ, тем выше эффективность прикрытия [12]. Таким образом, поддерживаемые ИП параметры боевого порядка определяются из соображений максимальной близости ИП к УИ и ограничиваются безопасностью полетов.

Истребитель ИО с работающей на излучение БРЛС, с одной стороны, должен формировать качественные оценки параметров движения ВЦ противника для выдачи их УИ, с другой - в интересах скрытия от БРЛС противника также может прикрываться помехами, создаваемыми ИП. Причем чем ближе истребитель ИО находится к ИП, тем выше эффективность прикрытия, но тем выше спектральная плотность мощности непреднамеренных помех, проникающих по боковым лепесткам диаграммы направленности антенны (ДНА), и, как следствие, ниже возможности по информационному обеспечению УИ. Исходя из вышеизложенного истребитель ИО должен осуществлять целенаправленное изменение своего положения в пространстве с учетом траектории полета ИП. В предположении равенства ДНА бортовой РЛС истребителя ИО и САП истребителя прикрытия и привязки ДНА к строительной оси самолета, что может быть, например, при работе БРЛС в режиме непрерывной пеленгации ВЦ, находящейся на большом удалениии, геометрия их взаимного расположения должна иметь следующие параметры [12]: $\beta$ - угол между линиями визирования ИП - истребитель противника и истребитель ИО - ИП должен составлять $90^{\circ}$; курсовые углы $\psi_{1}$ и $\psi_{2}$ должны быть равны, расстояние между ИП и истребителем ИО рассчитывается в соответствии с выражением

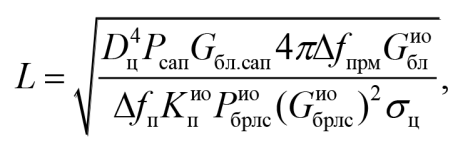

где $D_{\text {щ }}$ - требуемая дальность обнаружения воздушной цели; $P_{\text {сап }}-$ мощность помехи, излучаемая САП истребителя прикрытия; $G_{\text {бл.сап }}-$ коэффициент направленного действия боковых лепестков ДНА САП; $\Delta f_{\text {прм }}$ - полоса пропускания приемника БРЛС истребителя ИО; $G_{\sigma .}^{\text {ио }}-$ ко-

$$
-749-
$$


эффициент направленного действия боковых лепестков ДНА истребителя ИО; $\Delta f_{\text {п }}-$ ширина спектра помехи, излучаемой САП истребителя прикрытия; $K_{\text {по }}^{\text {ио }}$ коэффициент подавления

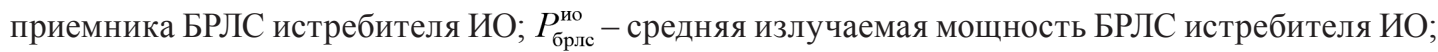
$\sigma_{ц}-$ эффективная поверхность отражения воздушной цели. Иными словами, боевой порядок «фронт» с заданным интервалом.

Как показал анализ публикаций [7-11, 13], посвященных наведению летательных аппаратов на ВЦ, в настоящее время отсутствуют методы наведения группы истребителей, учитывающих оптимальным образом характер решаемых тактических задач каждым истребителем в составе группы.

С учетом этого цель статьи - синтезировать метод наведения группы истребителей в составе ударного истребителя, истребителя прикрытия и истребителя информационного обеспечения на ВЦ, учитывающий тактическое назначение каждого истребителя в составе группы.

\section{Постановка задачи}

Весьма перспективным направлением синтеза сложных законов наведения, совместно наилучших по противоречивым требованиям точности и экономичности сигналов управления, является использование математического аппарата статистической теории оптимального управления в пространстве состояний.

Для группы истребителей в составе УИ, ИП и истребителя ИО, описываемых в векторноматричной форме выражением вида [6]

$$
\dot{\mathbf{x}}(t)=\mathbf{F} \mathbf{x}(t)+\mathbf{B u}(t)+\xi(t)
$$

где вектор состояния отдельного истребителя описывается моделью

$$
\begin{aligned}
& \dot{\mathbf{x}}_{i}(t)=\mathbf{F}_{i}(t) \mathbf{x}_{i}(t)+\mathbf{B}_{i}(t) \mathbf{u}_{i}(t)+\xi_{i}(t), i=1,2 \\
& \mathbf{F}_{1}=\mathbf{F}_{2}=\mathbf{F}_{3}, \quad \mathbf{F}_{i}=\left[\begin{array}{cc}
\mathbf{F}_{\mathrm{T} i} & 0 \\
0 & \mathbf{F}_{\mathrm{y}_{i}}
\end{array}\right], \quad \mathbf{B}_{i}=\left[\begin{array}{c}
0 \\
\mathbf{B}_{\mathrm{y}_{i}}
\end{array}\right]
\end{aligned}
$$

( $i=1$ соответствует ударному истребителю, $i=2$ - истребителю прикрытия, $i=3$ - истребителю информационного обеспечения) при наличии измерений,

$$
\mathbf{z}(t)=\mathbf{H x}(t)+\boldsymbol{\xi}_{\mathrm{u}}(t)
$$

необходимо сформировать управление $\mathbf{u}_{i}$ УИ, ИП и истребителем ИО, обеспечивающее минимум функционала качества

$$
\begin{aligned}
& I=M\left\{\mathbf{x}_{1}^{\mathrm{T}}(t) \mathbf{Q}_{1} \mathbf{x}_{1}(t)+\Delta \mathbf{x}_{12}^{\mathrm{T}}(t) \mathbf{G}_{1} \Delta \mathbf{x}_{12}(t)+\right. \\
& \left.+\Delta \mathbf{x}_{23}^{\mathrm{T}}(t) \mathbf{S}_{1} \Delta \mathbf{x}_{23}(t)+\int_{0}^{\mathrm{t}} \mathbf{u}_{i}^{\mathrm{T}}(t) \mathbf{K}_{i} \mathbf{u}_{i}(t) d t\right\} .
\end{aligned}
$$

В данном представлении $\quad \mathbf{x}=\left[\mathbf{x}_{1}^{\mathrm{T}} \mathbf{x}_{2}^{\mathrm{T}} \mathbf{x}_{3}^{\mathrm{T}}\right]^{\mathrm{T}}, \quad \xi=\left[\xi_{1}^{\mathrm{T}} \xi_{2}^{\mathrm{T}} \xi_{3}^{\mathrm{T}}\right]^{\mathrm{T}}, \quad \Delta \mathbf{x}_{12}=\mathbf{x}_{1}-\mathbf{x}_{2}, \quad \Delta \mathbf{x}_{23}=\mathbf{x}_{2}-\mathbf{x}_{3}$, $\mathbf{x}_{i}=\left[\mathbf{x}_{\mathrm{T} i}^{\mathrm{T}} \mathbf{x}_{\mathbf{y}_{i}}^{\mathrm{T}}\right]^{\mathrm{T}}-$ обобщенный вектор состояния отдельного истребителя, в котором

$$
\dot{\mathbf{x}}_{T i}(t)=\mathbf{F}_{\mathrm{Ti}} \mathbf{x}_{\mathrm{T} i}(t)+\boldsymbol{\xi}_{\mathrm{T} i}(t), \mathbf{x}_{\mathrm{T} i}(0)=\mathbf{x}_{\mathrm{T} i 0}
$$

- закон изменения требуемых фазовых координат;

$$
-750-
$$




$$
\dot{\mathbf{x}} \mathrm{y}_{i}(t)=\mathbf{F}_{\mathrm{y}_{i}} \mathbf{x}_{\mathrm{y}_{i}}(t)+\mathbf{B}_{\mathrm{y}_{i}} \mathbf{u}_{\mathrm{y}_{i}}(t)+\xi_{\mathrm{y}_{i}}(t), \mathbf{x}_{\mathrm{y}_{i}}(0)=\mathbf{x}_{\mathrm{y}_{i} 0}
$$

- закон изменения текущих (управляемых) фазовых координат.

В выражениях (2)-(7) $\mathbf{x}_{\mathbf{y}}, \mathbf{x}_{\mathrm{Ti}}-$ векторы управляемых и требуемых фазовых координат в текущие моменты времени $t ; \mathbf{F}_{\mathrm{y} i}$ и $\mathbf{F}_{\mathrm{T} i}$-динамические матрицы, учитывающие внутренние связи процессов (6) и (7); $\mathbf{B}_{\mathbf{y}_{i}}-$ матрица эффективности сигналов управления $\mathbf{u}_{i} ; \mathbf{z}-$ вектор измерений; $\mathbf{H}=\left[\begin{array}{ccc}\mathbf{H}_{1} & \mathbf{0} & \mathbf{0} \\ \mathbf{0} & \mathbf{H}_{2} & \mathbf{0} \\ \mathbf{0} & \mathbf{0} & \mathbf{H}_{3}\end{array}\right]$ - матрица связи обобщенного вектора состояния и измерений; $\mathbf{Q}_{1}=\left[\begin{array}{cc}\mathbf{Q} & -\mathbf{Q} \\ -\mathbf{Q} & \mathbf{Q}\end{array}\right]$, $\mathbf{G}_{1}=\left[\begin{array}{cc}\mathbf{G} & -\mathbf{G} \\ \mathbf{- G} & \mathbf{G}\end{array}\right]$ и $\mathbf{S}_{1}=\left[\begin{array}{cc}\mathbf{S} & -\mathbf{S} \\ \mathbf{- S} & \mathbf{S}\end{array}\right]$ - матрицы штрафов за точность наведения ударного истребителя, поддержания требуемой геометрии взаимного расположения ИП относительно УИ и поддержания требуемой геометрии взаимного расположения истребителя ИО относительно ИП; $\mathbf{K}_{i}$ - матрица штрафов за величину сигналов управления, $\mathbf{K}_{\mathbf{1}}=\mathbf{K}_{2}=\mathbf{K}_{3} ; \mathbf{M}$ - математическое ожидание функционала (5) при условии, что имеются измерения (4); $\xi_{\text {и }}$, $\xi_{T i}, \xi_{\mathrm{y} i}$ - центрированные гауссовские возмущения; 0 - нулевая матрица.

На основании выводов теоремы разделения и статистической эквивалентности с учетом линейности модели (2), гауссовости возмущений $\xi_{\text {й }}, \xi_{T i}, \xi_{\text {у }}$, квадратичности функционала (5) оптимальный закон управления может быть синтезирован независимо от формирователя оценок. В связи с этим в дальнейшем представлении все возмущения отсутствуют $\xi_{\text {и }}=\xi_{\mathrm{T} i}=\xi_{\mathrm{y} i}=0$, а значения фазовых координат заменяются их оптимальными оценками.

Для отыскания сигнала управления в условиях линейности модели, квадратичности функционала, гауссовости шумов может быть использовано уравнение Беллмана. Решением данного уравнения является оптимальный закон управления, минимизирующий функционал (5) вида [13]:

$$
\begin{aligned}
& \mathbf{u}_{i}=-\mathbf{K}_{i}^{-1} \mathbf{B}_{i}^{\mathrm{T}}\left(\mathbf{Q}_{1} \hat{\mathbf{x}}_{1}+\mathbf{G}_{1} \Delta \hat{\mathbf{x}}_{12}+\mathbf{S}_{1} \Delta \hat{\mathbf{x}}_{23}\right)=-\mathbf{K}_{i}^{-1}\left[\begin{array}{c}
0 \\
\mathbf{B}_{\mathrm{y}_{i}}
\end{array}\right]^{\mathrm{T}}\left\{\left[\begin{array}{ll}
\mathbf{Q} & -\mathbf{Q} \\
-\mathbf{Q} & \mathbf{Q}
\end{array}\right]\left[\begin{array}{l}
\hat{\mathbf{x}}_{\mathrm{T} 1} \\
\hat{\mathbf{x}}_{\mathrm{y} 1}
\end{array}\right]+\right. \\
& \left.+\left[\begin{array}{cc}
\mathbf{G} & -\mathbf{G} \\
\mathbf{- G} & \mathbf{G}
\end{array}\right]\left[\begin{array}{l}
\Delta \hat{\mathbf{x}}_{\mathrm{T} 12} \\
\hat{\mathbf{x}}_{\mathrm{y} 12}
\end{array}\right]+\left[\begin{array}{cc}
\mathbf{S} & -\mathbf{S} \\
\mathbf{- S} & \mathbf{S}
\end{array}\right]\left[\begin{array}{c}
\Delta \hat{\mathbf{x}}_{\mathrm{T} 23} \\
\Delta \hat{\mathbf{x}}_{\mathrm{y} 23}
\end{array}\right]\right\}= \\
& =\mathbf{K}_{1}^{-1} \mathbf{B}_{\mathrm{y} 1} \mathbf{Q}\left(\hat{\mathbf{x}}_{\mathrm{T} 1}-\hat{\mathbf{x}}_{\mathrm{y} 1}\right)+\mathbf{K}_{2}^{-1} \mathbf{B}_{\mathrm{y} 2} \mathbf{G}\left(\Delta \hat{\mathbf{x}}_{\mathrm{T} 12}-\Delta \hat{\mathbf{x}}_{\mathrm{y} 12}\right)+\mathbf{K}_{3}{ }^{-1} \mathbf{B}_{\mathrm{y} 3} \mathbf{S}\left(\Delta \hat{\mathbf{x}}_{\mathrm{T} 23}-\Delta \hat{\mathbf{x}}_{\mathrm{y} 23}\right) .
\end{aligned}
$$

Из (8) следует, что для ударного истребителя сигнал управления имеет вид

$$
\mathbf{u}_{1}=\mathbf{K}_{1}^{-1} \mathbf{B}_{\mathrm{y} 1} \mathbf{Q}\left(\hat{\mathbf{x}}_{\mathrm{T} 1}-\hat{\mathbf{x}}_{\mathrm{y} 1}\right)
$$

для истребителя прикрытия:

$$
\mathbf{u}_{2}=\mathbf{K}_{2}{ }^{-1} \mathbf{B}_{\mathrm{y} 2} \mathbf{G}\left(\Delta \hat{\mathbf{x}}_{\mathrm{T} 12}-\Delta \hat{\mathbf{x}}_{\mathrm{y} 12}\right)
$$

а для истребителя информационного обеспечения: 


$$
\mathbf{u}_{3}=\mathbf{K}_{3}^{-1} \mathbf{B}_{\mathrm{y} 3} \mathbf{S}\left(\Delta \hat{\mathbf{x}}_{\mathrm{T} 23}-\Delta \hat{\mathbf{x}}_{\mathrm{y} 23}\right)
$$

Особенностью сигнала управления (8) является то, что он обеспечивает оптимизацию управления на каждый момент времени и не требует априорного знания временного интервала функционирования системы. Кроме того, закон управления (8) позволяет учитывать возможность наведения ударного истребителя в ЗВПР по требуемой траектории (выражение 9), учитывать расположение ударного самолета истребителем прикрытия при совместном их полете (выражение 10), а также расположение истребителя ИО (выражение 11) при решении им задач по формированию оценок параметров движения ВЦ противника относительно ИП в интересах осуществления скрытого полета.

\section{Синтез метода наведения группы истребителей с учетом решаемых ими тактических задач}

Для синтеза закона совместного управления УИ, ИП и истребителем ИО в качестве модели состояния управляемого объекта была принята система дифференциальных уравнений, позволяющая преобразовывать фазовые координаты абсолютного движения цели и истребителей в координаты относительного движения, вида $[9,11,13]$

$$
\begin{aligned}
& \dot{\varphi}_{i}=\omega_{i}+\frac{j_{i}-j_{u}}{\dot{\not}_{i}}, \varphi_{i}(0)=\varphi_{i 0} \\
& \dot{\omega_{i}}=-\frac{2 \dot{\not}_{i}}{\mu_{i}} \omega_{i}-\frac{j_{i}-j_{u}}{\mu_{i}}, \omega_{i}(0)=\omega_{i 0},
\end{aligned}
$$

кроме того, для УИ и ИП вследствие отсутствия на них возможности формирования оценок параметров относительного движения из-за выключенной БРЛС и осуществления командного наведения с истребителя ИО по курсу дополнительно используется соотношение

$$
\dot{\psi_{i}}=j_{i} / V_{i}, \psi_{i}(0)=\psi_{i 0},
$$

где $\varphi_{i}-$ бортовой пеленг воздушной цели с УИ, бортовой пеленг 1-й виртуальной точки с ИП, бортовой пеленг 2-й виртуальной точки с истребителя ИО;

$\omega_{i}-$ угловая скорость вращения линий визирования УИ-цель, ИП - 1-я виртуальная точка, истребитель ИО - 2-я виртуальная точка;

$Д_{i}, Д_{i}-$ дальность между УИ и воздушной целью, ИП с 1-й виртуальной точкой, истребителя ИО со 2-й виртуальной точкой и скорости их изменения соответственно;

$j_{i}$ - поперечное ускорение УИ, ИП и истребителя информационного обеспечения;

$j_{\text {ц }}$ поперечное ускорение воздушной цели;

$\psi_{i}-$ курс ИП и УИ;

$V_{i}$ - скорость наводимого истребителя (УИ, ИП).

Виртуальные точки используются для информационного обеспечения ИП и истребителя ИО.

Представив (5), (12), (13) в векторно-матричной форме, с учетом $\mathbf{K}_{\mathbf{1}}=\mathbf{K}_{\mathbf{2}}=\mathbf{K}_{\mathbf{3}}$ для УИ получим

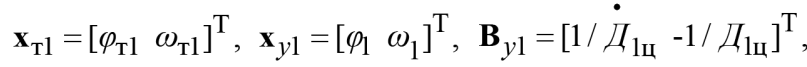

$$
\begin{aligned}
& -752-
\end{aligned}
$$




$$
\mathbf{Q}=\left[\begin{array}{cc}
q_{\varphi} & 0 \\
0 & q_{\omega}
\end{array}\right], \mathbf{K}_{1}=k_{1}, \mathbf{u}=\dot{\psi_{1}} V_{1}-j_{\text {Ц }}
$$

для ИП с учетом отсутствия зависимости сигналов управления от параметров движения

$$
\mathbf{x}_{\mathrm{T} 2}=\left[\begin{array}{ll}
\varphi_{\mathrm{T} 2} & \omega_{\mathrm{T} 2}
\end{array}\right]^{\mathrm{T}}, \quad \mathbf{x}_{y 2}=\left[\begin{array}{ll}
\varphi_{2} & \omega_{2}
\end{array}\right]^{\mathrm{T}}, \quad \mathbf{B}_{y 2}=\left[\begin{array}{ll}
1 & 1
\end{array}\right]^{\mathrm{T}}, \mathbf{G}=\left[\begin{array}{cc}
g_{\varphi} & 0 \\
0 & g_{\omega}
\end{array}\right], \mathbf{K}_{2}=k_{1},
$$

для истребителя ИО

$$
\mathbf{x}_{\mathrm{T} 3}=\left[\begin{array}{ll}
\varphi_{\mathrm{T} 3} & \omega_{\mathrm{T} 3}
\end{array}\right]^{\mathrm{T}}, \quad \mathbf{x}_{y 3}=\left[\begin{array}{ll}
\varphi_{3} & \omega_{3}
\end{array}\right]^{\mathrm{T}}, \mathbf{B}_{y 3}=\left[\begin{array}{ll}
1 & 1
\end{array}\right]^{\mathrm{T}}, \mathbf{S}=\left[\begin{array}{cc}
s_{\varphi} & 0 \\
0 & s_{\omega}
\end{array}\right], \quad \mathbf{K}_{3}=k_{1},
$$

где $q_{\varphi}, q_{\omega}$ - коэффициенты штрафов за точность выдерживания УИ требуемой траектории полета в ЗВПР класса «воздух-воздух»; $g_{\varphi}, g_{\omega}$ - коэффициенты штрафов за точность выдерживания ИП параметров боевого порядка; $s_{\varphi}, s_{\omega}$ - коэффициенты штрафов за точность выдерживания истребителем ИО параметров боевого порядка; $\varphi_{\mathrm{T} 1}, \omega_{\mathrm{T} 1}, \varphi_{\mathrm{T} 2}, \omega_{\mathrm{T} 2}, \varphi_{\mathrm{T} 3}, \omega_{\mathrm{T} 3}-$ требуемые значения пеленга и угловой скорости линии визирования УИ, ИП и истребителя ИО соответственно; $\not_{1 ц}, \dot{Д}_{1 ц}-$ дальность от УИ до цели и скорость ее изменения соответственно; $k_{1}-$ коэффициент штрафа за величину сигнала управления.

В интересах минимизации промаха при наведении ударного истребителя с учетом [13] $h \approx-\frac{Д_{1 щ}^{2} \omega_{1}}{\dot{म}_{1}}$ значение угловой скорости вращения линии визирования УИ $\omega_{\mathrm{T} 1}$ должно равняться 0 , т. е.

$$
\omega_{\mathrm{T} 1}=0 .
$$

Подставив (14) в (9), с учетом (13) и (17) получим закон изменения производной требуемого курса УИ в виде

$$
\left.\dot{\psi}_{1 \mathrm{~T}}=\frac{1}{V_{1}}\left[\frac{q_{\varphi}}{\hat{.}_{k_{1}}}\left(\hat{\varphi}_{\mathrm{Tl}}-\hat{\varphi}_{1}\right)+\frac{q_{\omega}}{k_{1} \hat{\partial}_{1 ц}} \hat{\omega}_{1}+\hat{j}_{\mathrm{L}}\right)\right],
$$

где $\hat{厶}_{1 ц}, \dot{\not}_{1 ц}-$ оценки дальности от УИ до цели и скорости ее изменения соответственно; $\varphi_{1}, \omega_{1}$ - оценки бортового пеленга на цель с УИ и угловой скорости вращения линии визирования УИ-ВЦ; $\hat{j}_{\text {ц }}$ - оценка поперечного ускорения ВЦ, причем [7] $\hat{\varphi}_{\mathrm{T} 1}=\arcsin \left(\frac{\hat{\not}_{1 Ц} \hat{\omega}_{1}+\hat{V}_{ц} \sin \hat{\varphi}_{1}}{\hat{V}_{1}}\right),\left|\hat{म}_{1 ц} \hat{\omega}_{1}+\hat{V}_{\text {ц }} \sin \hat{\varphi}_{1}\right| \leq \hat{V}_{\mathrm{c}}$, где $\hat{V}_{\text {щL }}$ - оценка скорости полета цели.

Поскольку информация о требуемых значениях курса УИ с истребителя ИО передается дискретно с периодом Т, обусловленным скоростью обмена информацией между ними, в дискретном представлении закон формирования требуемого значения курса определяется рекуррентным соотношением вида 


$$
\begin{aligned}
& \psi_{1 \mathrm{~T}}(k)=\psi_{1 \mathrm{~T}}(k-l)+\frac{\mathrm{T}}{V_{1}}\left[\frac{q_{\varphi}}{\hat{.}_{k_{1}}(k-l)}\left(\hat{\varphi}_{\mathrm{Tl}}(k-l)-\hat{\varphi}_{1}(k-l)\right)+\right. \\
& \left.\left.+\frac{q_{\omega}}{k_{1} \hat{Д}_{1 \mathrm{~L}}(k-l)} \hat{\omega}_{1}(k-l)+\hat{j}_{\mathrm{L}}(k-I)\right)\right]
\end{aligned}
$$

где $\psi_{1 \mathrm{~T}}(k)$ и $\psi_{1 \mathrm{~T}}(k-1)$ - требуемые значения курса УИ в k-й и $(\mathrm{k}-1)$-й моменты времени, отстоящие друг от друга на интервал времени $\mathrm{T}$.

Для реализации скрытого наведения УИ с выключенной его БРЛС на истребителе ИО в интересах формирования требуемого курса (19) по измеренным значениям дальностей от истребителя ИО до ВЦ Дзц и от истребителя ИО до УИ Д ${ }_{31}$, а также углов визирования $\varepsilon_{3 ц}$ ВЦ

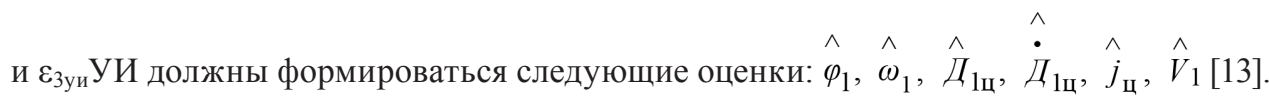

Для ИП в интересах поддержания боевого порядка, определяемого минимальным расстоянием между УИ и ИП, с учетом безопасности полета вектор требуемых фазовых координат имеет вид

$$
\hat{\varphi}_{\mathrm{T} 2}=\hat{\varphi}_{\mathrm{T} 1}, \hat{\omega}_{\mathrm{T} 2}=\hat{\omega}_{\mathrm{T} 1}=0
$$

Подставив (15) в (10), с учетом (13) и (20) получим закон изменения производной требуемого курса ИП, формируемого на истребителе ИО в виде

$$
\dot{\psi}_{2 \mathrm{~T}}=\frac{1}{V_{2}}\left[\frac{q_{\varphi}}{k_{1}}\left(\Delta \hat{\varphi}_{\mathrm{T} 12}-\Delta \hat{\varphi}_{\mathrm{y} 12}\right)+\frac{q_{\omega}}{k_{1}}\left(\Delta \hat{\omega}_{\mathrm{T} 12}-\Delta \hat{\omega}_{\mathrm{y} 12}\right)\right] .
$$

В дискретном представлении (21) имеет вид

$$
\begin{aligned}
& \psi_{2 \mathrm{~T}}(k)=\psi_{2 \mathrm{~T}}(k-l)+ \\
& +\frac{\mathrm{T}}{V_{2}}\left[\frac{q_{\varphi}}{k_{1}}\left(\Delta \hat{\varphi}_{\mathrm{T} 12}-\Delta \hat{\varphi}_{\mathrm{Y} 12}\right)+\frac{q_{\omega}}{k_{1}}\left(\Delta \hat{\omega}_{\mathrm{T} 12}-\Delta \hat{\omega}_{\mathrm{y} 12}\right)\right], \\
& \Delta \hat{\varphi}_{\mathrm{T} 12}=\hat{\varphi}_{\mathrm{T} 1} \hat{\varphi}_{\mathrm{T} 2} \Delta \hat{\omega}_{\mathrm{T} 12}=\hat{\omega}_{\mathrm{T} 1}-\hat{\omega}_{\mathrm{T} 2} \Delta \hat{\varphi}_{\mathrm{Y} 12}= \\
& =\hat{\varphi}_{\mathrm{y} 1} \hat{\varphi}_{\mathrm{y} 2} \Delta \hat{\omega}_{\mathrm{y} 12}=\hat{\omega}_{\mathrm{y} 1}-\hat{\omega}_{\mathrm{y} 2},
\end{aligned}
$$

где $\hat{\varphi}_{\mathrm{y} 1}-$ оценки текущих значений пеленгов ВЦ с ударного истребителя; $\hat{\varphi}_{\mathrm{y} 2}-$ оценки текущих значений пеленгов 1-й виртуальной точки с истребителя прикрытия, положение виртуальной точки меняется в процессе полета воздушной цели и определяется положением воздушной цели и расстоянием, обеспечивающим безопасность наведения; $\hat{\omega}$ у1, $\hat{\omega} 2$ - оценки текущих значений угловых скоростей вращения линий визирования «ударный истребитель-воздушная цель» и «истребитель прикрытия - 1-я виртуальная точка». 
Для осуществления наведения ИП с выключенной БРЛС и включенной САП на истребителе ИО в интересах формирования требуемого курса (22) по измеренным значениям дальностей

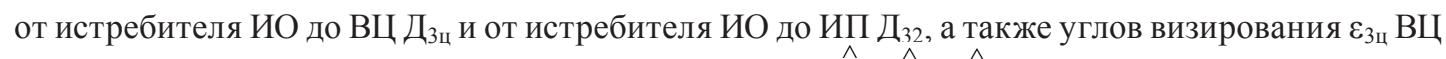

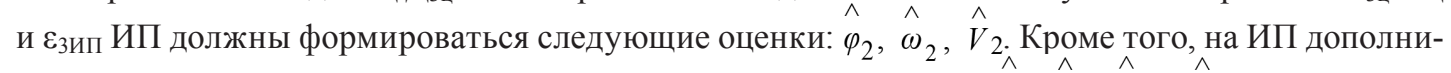
тельно с истребителя ИО необходимо передавать информацию о $\hat{\varphi}_{1}, \hat{\omega}_{1}, \hat{\varphi}_{\mathrm{Tl}}, \hat{\omega}_{\mathrm{Tl}}$.

Для наведения истребителя ИО, осуществляющего поддержание параметров боевого порядка относительно ИП, описанных во введении, вектор требуемых фазовых координат имеет вид

$$
\hat{\varphi}_{\mathrm{T} 3}=\hat{\varphi}_{\mathrm{T} 2}, \hat{\omega}_{\mathrm{T} 3}=\hat{\omega}_{\mathrm{T} 2}=0
$$

Подставив (16) в (11), с учетом (23) получим закон управления поперечной перегрузкой истребителя ИО в виде

$$
\begin{aligned}
& j_{3}=\frac{q_{\varphi}}{k_{1}}\left(\Delta \hat{\varphi}_{\mathrm{T} 23}-\Delta \hat{\varphi}_{\mathrm{y} 23}\right)+\frac{q_{\omega}}{k_{1}}\left(\Delta \hat{\omega}_{\mathrm{T} 23}-\Delta \hat{\omega}_{\mathrm{y} 23}\right), \\
& \Delta \hat{\varphi}_{\mathrm{T} 23}=\hat{\varphi}_{\mathrm{T} 2}-\hat{\varphi}_{\mathrm{T} 3} \Delta \hat{\omega}_{\mathrm{T} 23}=\hat{\omega}_{\mathrm{T} 2}-\hat{\omega}_{\mathrm{T} 3} \Delta \hat{\varphi}_{\mathrm{y} 23}= \\
& =\hat{\varphi}_{\mathrm{y} 2}-\hat{\varphi}_{\mathrm{y} 3} \Delta \hat{\omega}_{\mathrm{y} 23}=\hat{\omega}_{\mathrm{y} 2}-\hat{\omega}_{\mathrm{y} 3},
\end{aligned}
$$

где $\hat{\varphi}_{\text {уз }}$ - оценка текущего значения пеленга 2-й виртуальной точки с истребителя ИО, положение 2-й виртуальной точки определяется положением 1-й виртуальной точки и расстоянием $\mathrm{L}$ по фронту; $\hat{\omega}$ у - оценка текущего значения угловой скорости вращения линии визирования «истребитель ИО - 2-я виртуальная точка».

Для осуществления наведения истребителя ИО на нем в интересах формирования требуемой перегрузки (24) по измеренным значениям дальностей от истребителя ИО до ВЦ Дзц

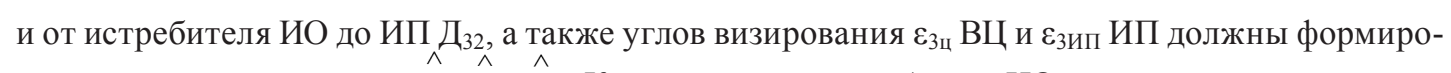
ваться следующие оценки: $\hat{\varphi}_{2}, \hat{\omega}_{2}, \hat{V}_{2}$. Кроме того, на истребителе ИО дополнительно на основе измеренных значений пеленга 2-й виртуальной точки с истребителя ИО, угловой скорости вращения линии визирования «истребитель ИО - 2-я виртуальная точка» и описанных ранее параметров, используемых для вычисления $\hat{\varphi}_{\mathrm{Tl}}$, должны формироваться соответствующие оценки.

На основе результатов проведенного анализа следует отметить, что информационное обеспечение разработанных алгоритмов (19), (22), (24) может быть произведено по существующим измерителям, что свидетельствует о возможности их практической реализации.

\section{Результаты моделирования}

Исследование предложенных алгоритмов (19), (22,) (24) проводилось с помощью пакета инженерных вычислений MATLAB в процессе моделирования полета воздушной цели и наводимой на нее группы в составе УИ, ИП и истребителя ИО.

Кроме того, для информационного обеспечения истребителей группы при их наведении, в частности ИП и истребителя ИО, при формировании оценок текущих пеленгов и угловых скоростей линий визирования моделировались эволюции двух виртуальных точек, изменяющих свое положение в пространстве, в зависимости от положения цели, а также расстояниями УИ-ИП и ИП-истребитель ИО.

$$
-755-
$$


В качестве показателей эффективности предложенных алгоритмов для УИ принят текущий промах, вычисляемый в соответствии с выражением $h=\hat{\not}_{1 \Perp}^{2} \hat{\omega} \hat{y}_{1} / \hat{\not}_{1 ц}$, для истребителя прикрытия - реализуемый на входе приемника БРЛС истребителя противника коэффициент подавления $K_{\Pi}^{\text {прот }}=\frac{P_{\Pi}^{\text {прот }}}{P_{\mathrm{c}}^{\text {прот }}}=\frac{4 \pi P_{\text {сап }} G_{\text {о.сап }} \Delta f_{\text {прм }}^{\text {прот }} \hat{Д}_{1 щ}^{2}}{\Delta f_{\Pi} P_{\text {брот }}^{\text {прот }} G_{\text {о.брот }}^{\text {прл }} \sigma_{\text {и }}}$, где кроме ранее принятых $P_{\Pi}^{\text {прот }}-$ мощность помехи на входе приемника БРЛС противника; $P_{\mathrm{c}}^{\text {прот }}$ - мощность сигнала на входе приемника

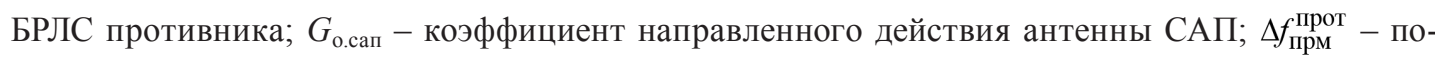

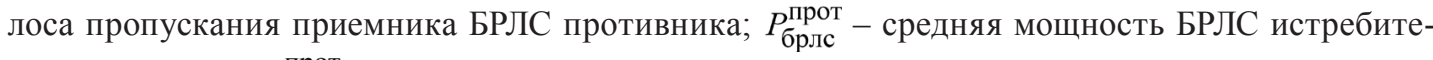
ля противника; $G_{\text {о.брлс }}^{\text {прот }}$ коэффициент направленного действия антенны БРЛС противника; $\sigma_{\text {и }}$ - эффективная поверхность рассеивания истребителя. Для истребителя ИО - реализуемый коэффициент подавления на входе приемника его БРЛС, поддерживаемый в результате обеспечения им требуемого боевого порядка, вычисляемый в соответствии с выражением

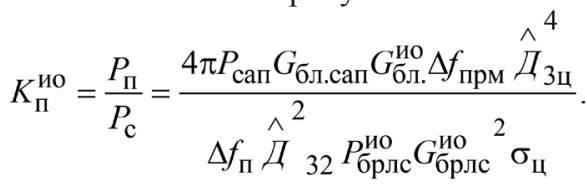

Для расчетов и моделирования были использованы следующие исходные данные: $P_{\text {сап }}=200$ Вт; $G_{\text {бл.сап }}=2 ; \Delta f_{\text {прм }}=100$ Гц; $G_{\text {бл }}^{\text {ио }}=4 ; \Delta f_{\text {п }}=50$ МГц; $K_{\text {пा }}^{\text {но }}=4 ; P_{\text {брлс }}^{\text {ио }}=P_{\text {брлс }}^{\text {про }}=2500$ Вт; $G_{\text {брлс }}^{\text {ио }}=G_{\text {брлс }}^{\text {прог }}=10^{4} ; \sigma_{\text {ц }}=\sigma_{\text {и }}=5 \mathrm{~m}^{2} ; \Delta f_{\text {п }}=50$ МГц, $\Delta f_{\text {прм }}=\Delta f_{\text {прм }}^{\text {прот }}=100$ Гц, $D_{\text {ц }}=150$ км. Рассчитанное в соответствии с выражением (1) значение $L=1427$ м. Исходя из приведенных исходных данных пороговое значение реализуемого коэффициента подавления БРЛС истребителя ИО $K_{\pi}^{\text {ио }}=4$. В предположении равенства потенциальных характеристик БРЛС конфликтующих сторон для истребителя противника пороговое значение реализуемого коэффициента подавления БРЛС истребителя принято 4.

Результаты моделирования наведения истребителей на рис. 1, 2 изображены в декартовой системе координат. На данных рисунках принято: 1 - траектория УИ, 2 - траектория ИП, 3 траектория истребителя ИО, 4 - траектория ВЦ, 5 - траектория 1-й виртуальной точки для информационного обеспечения ИП, 6 - траектория 2-й виртуальной точки для информационного обеспечения истребителя ИО.

\section{Начальные условия моделирования траекторий полетов самолетов}

Противник: 1) положение ВЦ $\mathrm{x}_{0 Ц}=0 \mathrm{M}, \mathrm{z}_{0 Ц}=150000$ м. 2) С учетом требований безопасности полета расстояние между УИ и ИП определено 500 м, т. о., начальные координаты 1-й виртуальной точки (траектория 5, рис. 1) $\mathrm{x}_{0 \mathrm{BT1}}=-500$ м, $\mathrm{z}_{0 \mathrm{BT1}}=150000$ м. 3) С учетом L начальные координаты 2-й виртуальной точки, используемые при наведении истребителя прикрытия, $\mathrm{x}_{0 \mathrm{BL}}=-1927$ м, $\mathrm{z}_{0 \mathrm{BL}}=150000$ м. 4) Начальные курсы ВЦ и виртуальных точек равны $\psi_{0 \mathrm{Bц}}=\psi_{0 \mathrm{BT1}}=$ $=\psi_{0 \text { вт2 }}=268^{\circ}$. Скорость полета ВЦ и виртуальных точек принята $200 \mathrm{M} / \mathrm{c}$.

Группа истребителей: 1) ударный истребитель: $\left.x_{0 у и}=-2000 \mathrm{M}, z_{0 у и}=0 \mathrm{м}, \psi_{0 у и}=0^{0} ; 2\right)$ истребитель прикрытия: $x_{0 и п}=-2500 \mathrm{M}, z_{0 у и}=0 \mathrm{M}, \psi_{0 у и}=0^{0}$; 3) истребитель информационного обеспечения: с учетом L $x_{0 \text { ио }}=-3927$ м, $z_{0 и о}=0$ м, $\psi_{0 и о ~}=0^{0}$. Скорость полета группы принята $250 \mathrm{M} / \mathrm{c}$. 
На рис. 1 приведены изменения взаимного расположения ВЦ и наводимой на нее группы истребителей при отсутствии маневра цели, на рис. 2 - изменения взаимного расположения ВЦ и наводимой на нее группы истребителей при маневрировании ВЦ.

Анализ рис. 1 и 2 свидетельствует о возможности при реализации алгоритмов наведения (19), (22,) (24) перехвата УИ воздушной цели, а также поддержание требуемых параметров боевого порядка ИП и истребителем ИО как при маневрировании ВЦ, так и при отсутствии маневра.

На рис. 3, 4 представлены оценки изменения текущего промаха ударного истребителя для ситуаций отсутствия маневра ВЦ (траектории рис. 1) и при ее маневрировании соответственно (траектории рис. 2).

Анализ рис. 3 и 4 свидетельствует, что в процессе наведения текущий промах УИ независимо от маневрирования цели стремится к нулю.

На рис. 5, 6 отражены оценки расстояния между ИП и истребителем ИО, значения мощности помехи, создаваемой ИП на входе приемника БРЛС истребителя ИО и реализуемо-

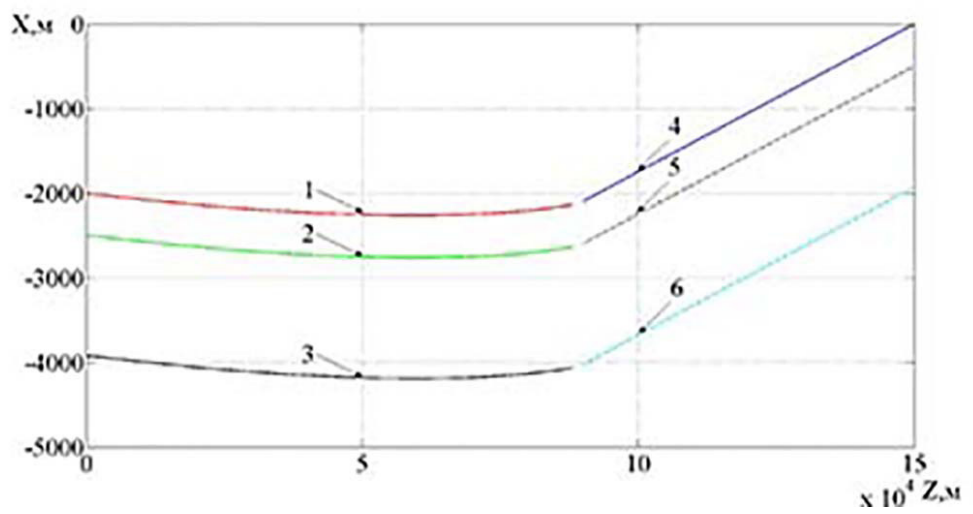

Рис. 1. Траектории полета группы при отсутствии маневра цели

Fig. 1. Flight paths of the group in the absence of a target maneuver

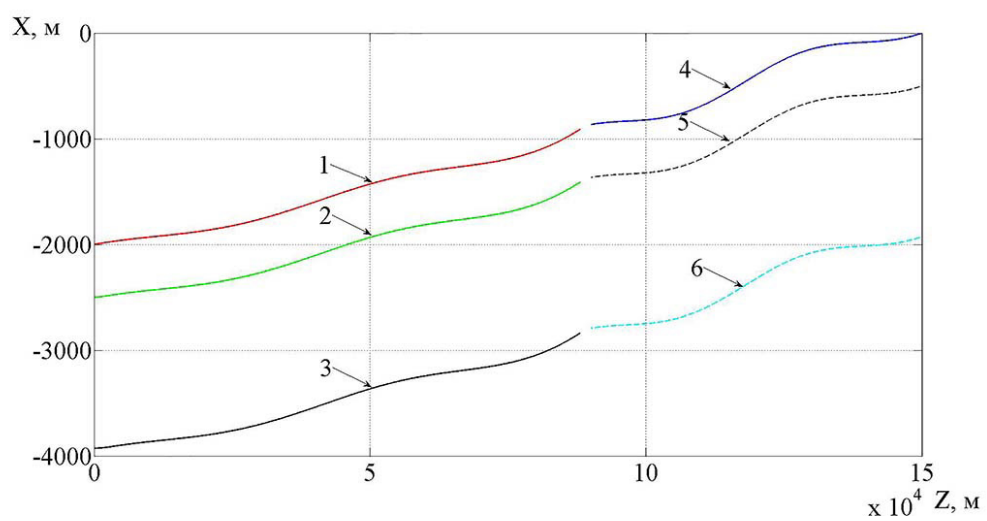

Рис. 2. Траектории полета группы при маневрировании цели

Fig. 2. Flight paths of the group when maneuvering the target 


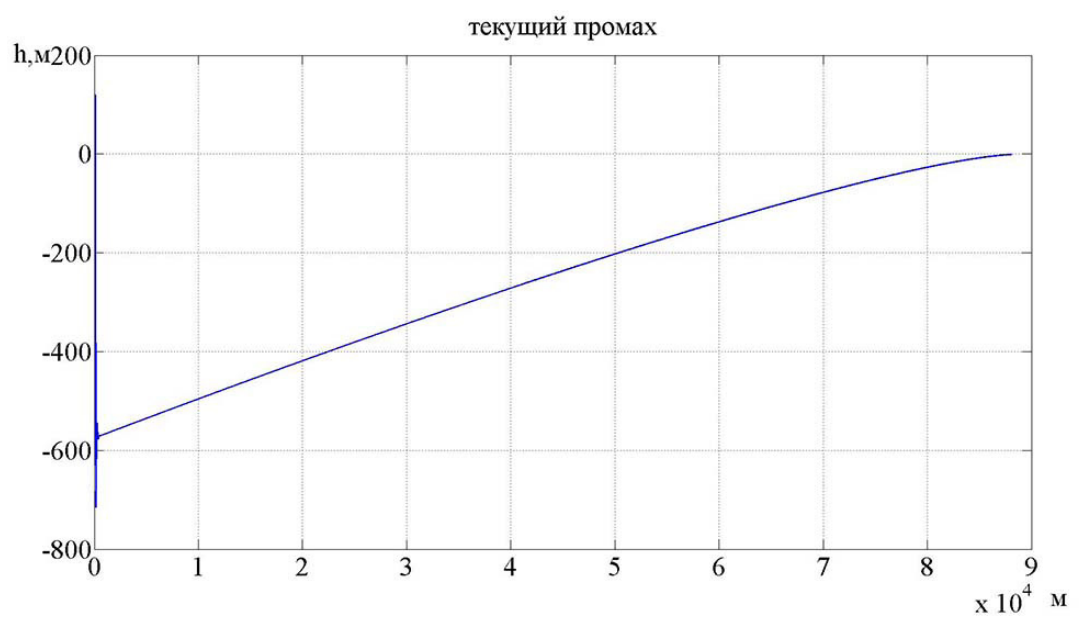

Рис. 3. Оценка текущего промаха при отсутствии маневра ВЦ

Fig. 3. Assessment of the current miss in the absence of an air target maneuver

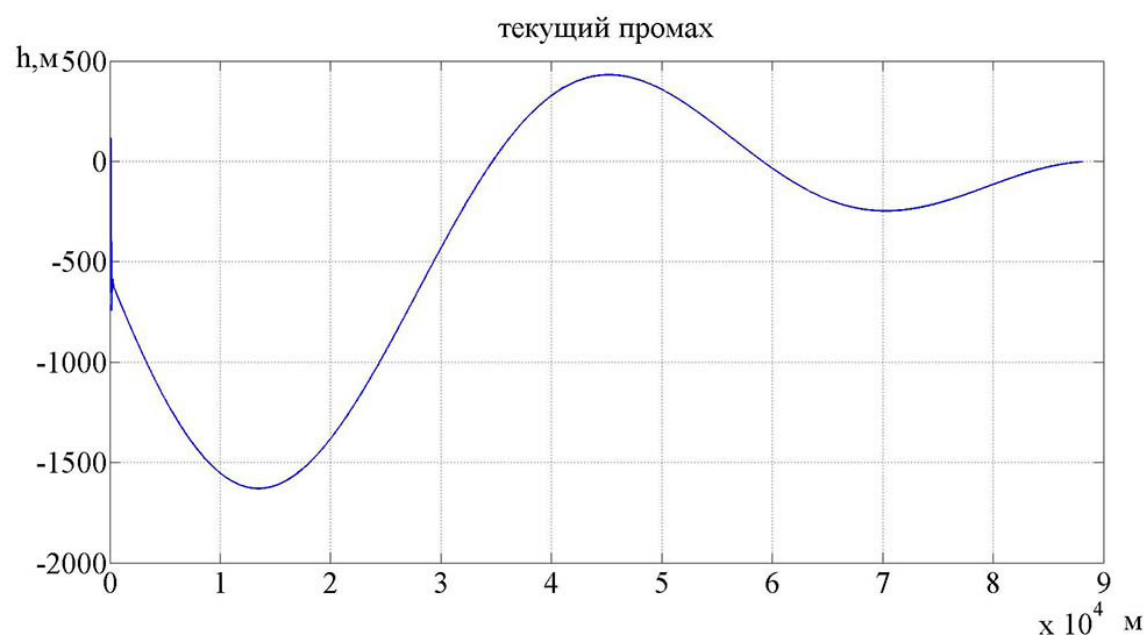

Рис. 4. Оценка текущего промаха при маневрировании ВЦ

Fig. 4. Assessment of the current miss when maneuvering an aerial target

го на входе приемника БРЛС истребителя ИО коэффициента подавления, причем на рис. 5 для ситуации отсутствия маневра цели (траектории рис. 1), на рис. 6 - ситуация маневрирования цели (траектории рис. 2). Анализ данных зависимостей позволяет заключить, что в процессе наведения происходят незначительные колебания расстояния между истребителем ИО и ИП, что, в свою очередь, приводит к незначительному колебанию уровня мощности непреднамеренной помехи, создаваемой САП истребителя прикрытия на входе приемника БРЛС ударного истребителя. Однако данные колебания несущественны. Оценка реализуемого коэффициента подавления свидетельствует о том, что при поддержании в соответствии с разработанным методом наведения параметров боевого порядка в процессе сближения с воздушной целью данный коэффициент не превосходит порогового значения, 


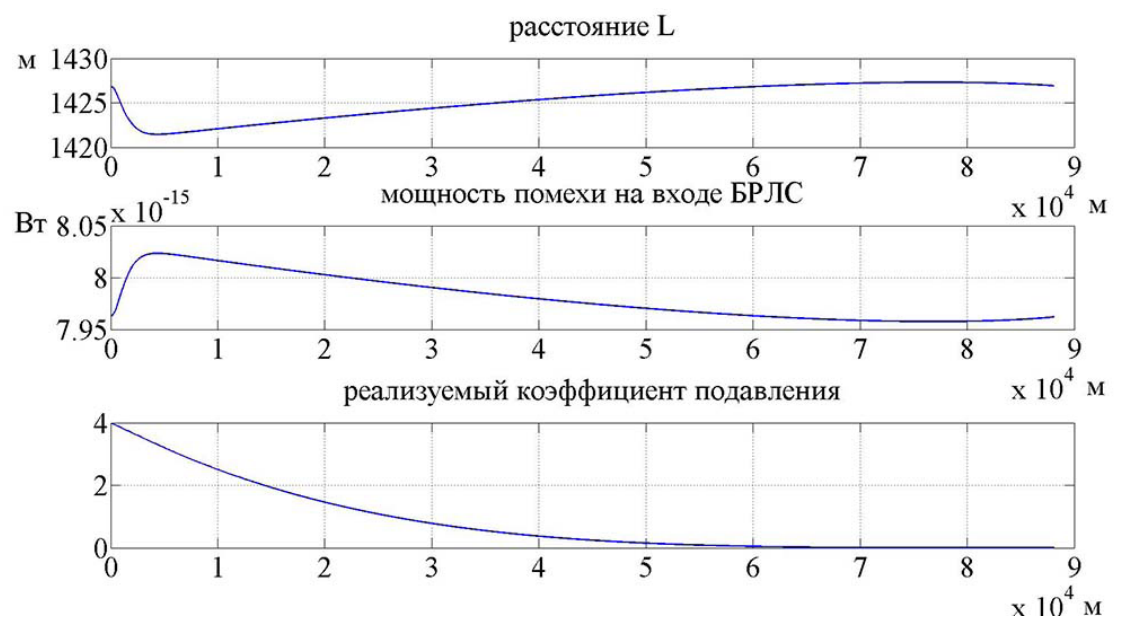

Рис. 5. Оценка реализуемого коэффициента подавления на входе приемника БРЛС истребителя ИО при отсутствии маневра

Fig. 5. Evaluation of the implemented suppression coefficient at the input of the radar receiver of the information support fighter in the absence of an air target maneuver

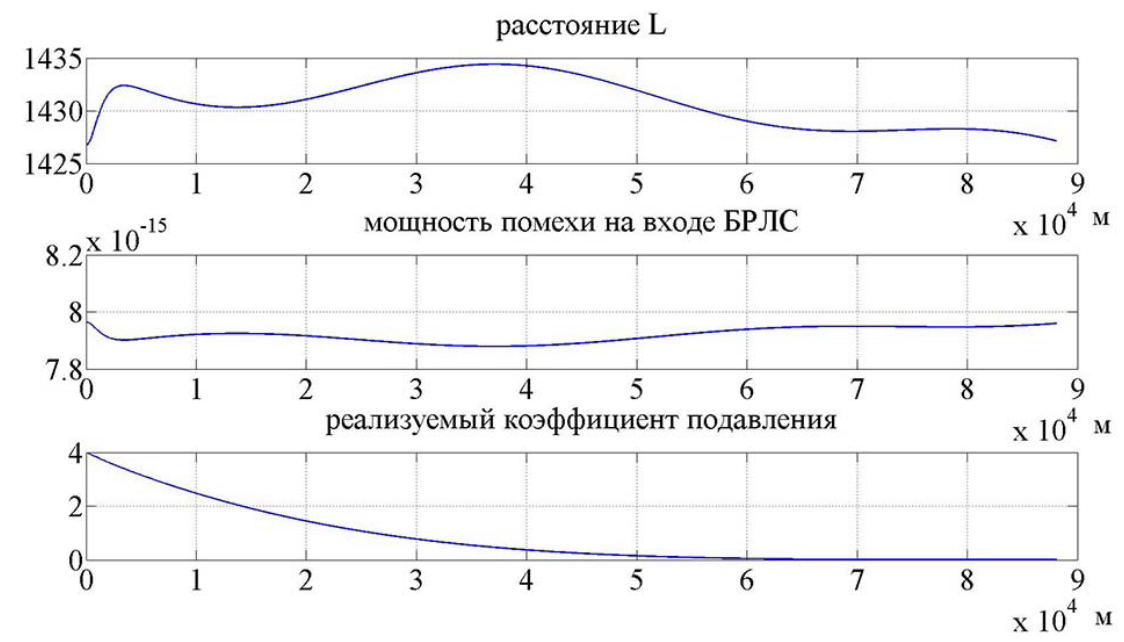

Рис. 6. Оценка реализуемого коэффициента подавления на входе приемника БРЛС истребителя ИО при маневрировании ВЦ

Fig. 6. Evaluation of the implemented suppression coefficient at the input of the radar receiver of the fighter information support when maneuvering an air target

при котором нарушается эффективная работа БРЛС (для рассматриваемого примера коэффициент подавления $\left.K_{\text {пI }}^{\text {но }}=4\right)$.

На рис. 7 представлена зависимость реализуемого коэффициента подавления на входе приемника БРЛС истребителя противника, соответствующая ситуациям полета ВЦ без маневра и при маневрировании.

Анализ рис. 7 свидетельствует о том, что в процессе всего наведения реализуемый коэффициент подавления, создаваемый на входе БРЛС истребителя противника, превышает пороговое значение 4, что свидетельствует о неустойчивой работе его БРЛС. 


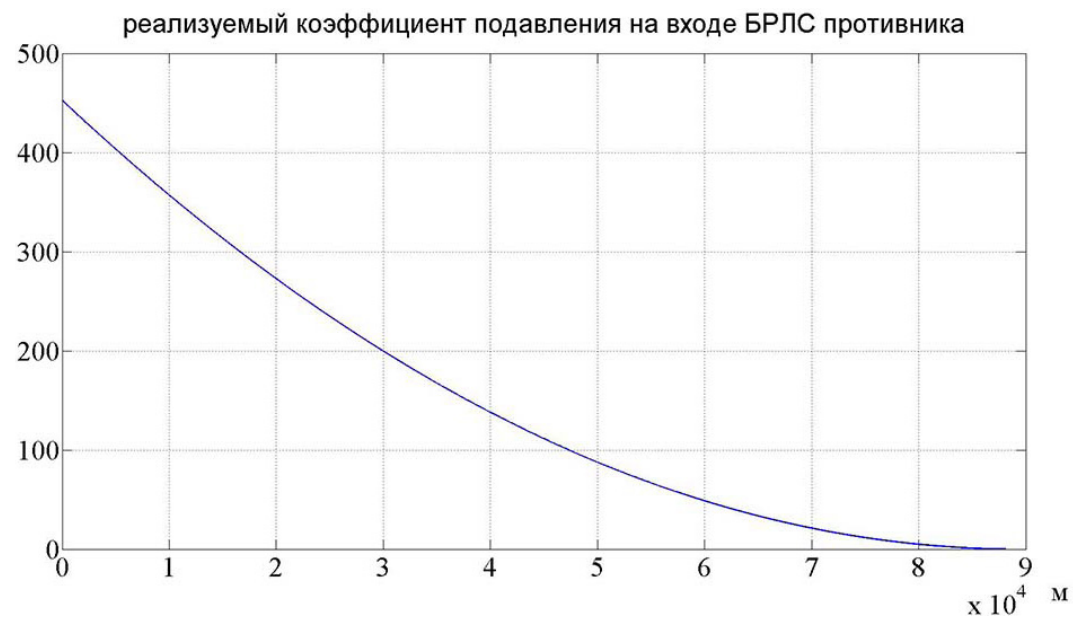

Рис. 7. Оценка реализуемого коэффициента подавления на входе приемника БРЛС противника

Fig. 7. Evaluation of the implemented suppression coefficient at the input of the enemy radar receiver

Таким образом, в данной статье поставлена и решена задача синтеза метода наведения группы истребителей в составе ударного истребителя, истребителя прикрытия и истребителя информационного обеспечения на ВЦ, учитывающего тактическое назначение каждого истребителя в составе группы.

\section{Заключение}

1. Весьма перспективным способом повышения эффективности групповых действий истребителей является распределение тактических задач между истребителями в составе группы (определение УИ, ИП и истребителя ИО) и наведение их с учетом решаемых задач.

2. Разработанный метод наведения позволяет осуществить скрытое точное наведение ударного истребителя в ЗВПР класса «воздух-воздух» за счет прикрытия его ИП, что позволяет противодействовать средствам активной локации и информационного обеспечения истребителем ИО, а это дает возможность противодействовать средствам пассивной локации ввиду выключенной БРЛС УИ.

3. Оценка эффективности синтезированного метода наведения группы истребителей позволила установить, что УИ наводится в ЗВПР класса «воздух-воздух» с высокой точностью, ИП при поддержании параметров позволяет эффективно подавлять БРЛС противника, истребитель ИО в процессе наведения за счет поддержания требуемых параметров боевого порядка с ИП, наряду с противодействием средствам активной локации противника, обеспечивает устойчивую работу его БРЛС.

\section{Список литературы / References}

[1] Веселов В., Фененко А. «Воздушная мощь» в мировой политике. Международные nроцессы. 2016, 14(3), 6-27. [Veselov V., Fenenko A. «Air power» in world politics. International processes. 2016, 14(3), 6-27 (in Russian)] 
[2] Кольцов Ю.В., Добычина Е. М. Авионика многофункционального истребителя Су-35. Успехи современной радиоэлектроники. 2019, 73(4), 31-48 [Kolcov J. V., Dobichina E. M. Avionics of the Su-35 multifunctional fighter. Advances in modern radio electronics. 2019, 73(4), 31-48 (in Russian)]

[3] Кольцов Ю. В., Добычина Е. М. Авионика истребителя пятого поколения Су-57. Усnехи современной радиоэлектроники. 2019, 73(8), 29-45 [Kolcov J.V., Dobichina E. M. Avionics of the fifth-generation Su-57 fighter. Advances in modern radio electronics. 2019, 73(8), 29-45 (in Russian)]

[4] Метод повымения помехозащищинности бортовой радиолокаџионной станщии истребителя: монография. А. В. Богданов [и др.], рук. авт. коллектива Д. В. Закомолдин. Красноярск: Сиб. федер. ун-т, 2018. 182 с. [The method of increasing the noise immunity on-board fighter radar: monograph A. V. Bogdanov [and others], the head of copyright. collective D. V. Zakomoldin. Krasnoyarsk: Siberian Federal University, 2018 (in Russian)]

[5] Богданов А.В., Закомолдин Д.В., Голубенко В.А., Кочетов И.В., Кучин А.А., Акимов С.И. Способ функиионирования импульсно-доплеровской бортовой радиолокаџионной станции истребителя при обеспечении энергетической скрытности еt работы на излучение. Патент на изобретение № 2694891 МПК G01S13/52 (2006.01), Россия, заявка № 2019104450, приоритет 18.02.2019, зарегестрировано 18.07.2019. Бюл № 20. [Bogdanov A. V., Zakomoldin D. V., Golubenko V.A., Kochetov I. V., Kuchin A. A., Akimov S. I. The method of functioning of the pulseDoppler onboard radar of a fighter while ensuring the energy stealth of its operation on radiation. Patent for invention No. 2694891 IPC G01S13/52 (2006.01), Russia, application No. 2019104450, priority 18.02.2019, registered 18.07.2019. Byul No. 20. (in Russian)]

[6] Богданов А.В., Закомолдин Д.В, Кочетов И.В., Акимов С.И. Методы противодействия средствам радиотехнической разведки противника при функционировании импульснодоплеровских бортовых РЛС истребителей на излучение. Успехи современной радиоэлектроники, 2021, 75(2). [Bogdanov A. V., Zakomoldin D. V., Kochetov I. V., Akimov S.I. Methods of counteraction to means of radio-technical intelligence of the enemy at functioning of pulseDoppler onboard radars of fighters on radiation. Advances in modern radio electronics, 2021, 75(2) (in Russian)]

[7] Авиационные системы радиоуправления. Т. 1. Принципы построения систем радиоуправления. Основы синтеза и анализа. Под ред. А.И. Канащенкова и В.И. Меркулова. М.: Радиотехника, 2003. 192 с. [Aviation radio control systems. Vol. 1. Principles of building radio control systems. Fundamentals of synthesis and analysis. Ed. by A.I. Kanashchenkov and V. I. Merkulov. M.: Radio Engineering, 2003. 192 p. (in Russian)]

[8] Авиационные системы радиоуправления. Т. 2. Радиоэлектронные системы самонаведения. Под ред. А.И. Канащенкова и В.И. Меркулова. М.: Радиотехника, 2003. 389 с. [Aviation radio control systems. Vol. 2. Radioelectronic homing systems. Ed. by A.I. Kanashchenkov and V. I. Merkulov. M.: Radio Engineering, 2003. 389 p. (in Russian)]

[9] Авиационные системы радиоуправления. Т. 3. Системы командного радиоуправления. Автономные и комбинированные системы наведения. Под ред. А. И. Канащенкова и В. И. Меркулова. М.: Радиотехника, 2004. 320 с [Aviation radio control systems. Vol. 3. Command radio control systems. Autonomous and combined guidance systems. Ed. by A.I. Kanashchenkov and V. I. Merkulov. M.: Radio Engineering, 2004. 320 p. (in Russian)]

$$
-761-
$$


[10] Информационно-измерительные и управляющие радиоэлектронные системы и комплексы. Монография. Под ред. В.С. Вербы. М.: Радиотехника, 2020. 490 с. [Information-measuring and control radio-electronic systems and complexes. Monograph. Edited by V.S. Verba. M.: Radio Engineering, 2020. 490 p. (in Russian)]

[11] Системы радиоуправления. Кн. 4. Оптимизачия алгоритмов управления. Кол. монография под ред. В.И. Меркулова. М.: Радиотехника, 2018. 312 с. [Radio control systems. Book 4. Optimization of control algorithms. Kol. Monograph edited by V. I. Merkulov. M.: Radio Engineering, 2018. 312 p. (in Russian)]

[12] Богданов А. В., Закомолдин Д.В. Метод наведения пары истребителей с распределением между ними задач подавления и поражения воздушной цели. Радиотехника. М.: Радиотехника, 2021. [Bogdanov A. V., Zakomoldin D. V. Method of guidance of a pair of fighters with the distribution between them of tasks of suppression and destruction of an air target. Radio Engineering. M.: Radio Engineering, 2021 (in Russian)]

[13] Комплексы с беспилотными летательными аппаратами. В 2 кн. Кн 2. Робототехнические комплексы на основе БЛА. Монография. Под ред. В.С. Вербы, Б. Г. Татарского. М. Радиотехника. 2016. 824 с. [Complexes with unmanned aerial vehicles. In $2 \mathrm{kn}$. Kn 2. Robotic systems based on UAVs. Monograph. Edited by V. S. Verba, B. G. Tatarsky. M. Radio Engineering. 2016. 824 p. (in Russian)] 